\title{
Control of the emission properties of organic light emitting diodes using plasmonic gratings of Ag nanoparticles
}

Sarah. Hamdad, Amadou. Thierno. Diallo, Mahmoud. Chakaroun, and Azzedine. Boudrioua

Laboratoire de Physique des Lasers CNRS UMR 7538, Université Paris 13, Sorbonne Paris Cité, Villetaneuse, France

\begin{abstract}
We report the influence of Ag nanoparticle arrays on the emission properties of organic light emitting diodes (OLED). First, we studied the features of Ag arrays related to the periodicity. The obtained results emphasize the existence of two coupling regimes dominated by near or far field effects, respectively. Thereafter, we studied the influence of these gratings on the performances of OLED devices. Our results reveal the benefit of the plasmonic structures which allow a precise tuning of the OLED properties.
\end{abstract}

\section{Introduction}

In recent years, several works have been reported on the plasmonic effect of nanoparticle arrays [1]. Besides, numerous studies concerning the interaction of metallic nanoparticles nearby active molecules have been, also, reported [2]. Nevertheless, a deep understanding of the influence of metallic nanoparticle gratings on the optical responses of organic emitters is still needed, in order to efficiently control their emission features. Many questions are still under investigation and need to be thoroughly studied. For instance, the plasmonic mode contribution to the diffractive orders has not been clarified. Moreover, almost the studies, particularly, focus on the optical excitation of emitter-nanoparticle system. Thereby, understanding this phenomenon under electrical excitation might reveal new coupling features and bring additional insight to the effects induced by plasmonic structures. The careful study of these processes are particularly crucial to develop efficient and new organic light emitting diodes (OLED), which may pave the way to new organic laser diode.

In this context, we report the investigation of the influence of $\mathrm{Ag}$ nanoparticle arrays on the emission of OLED devices. Specially, we aim to study the interaction range of plasmonic nanoparticles on the injection, recombination and extraction mechanisms of organic hetero-structures. For that purpose, we performed a study of the optical responses of $\mathrm{Ag}$ arrays by varying their periodicities in order to clarify the existence of two coupling regimes, from short periodicities dominated by near field effect to the longue ones exhibiting merely far field features. Thereafter, we studied the influence of these regimes on the radiative lifetimes of nearby emitters. Finally, the electrical and optical characteristics of OLEDs containing Ag nanoparticle arrays have been, carefully, studied.

\section{Results and discussion}

The studied structures are regular square arrays of silver nano-cylinders of $100 \mathrm{~nm}$ diameter on top of glass/ITO substrate, covered by $100 \mathrm{~nm}$ of organic material. Various $\mathrm{Ag}$ arrays with periods $(p)$ ranging from $180 \mathrm{~nm}$ to $480 \mathrm{~nm}$ by a step of $20 \mathrm{~nm}$, fabricated by using electron beam lithography technique, have been simulated and experimentally studied. For instance, fig.1.a shows the experimental map of the extinction intensity as a function of the $\mathrm{Ag}$ grating period at a normal excitation.

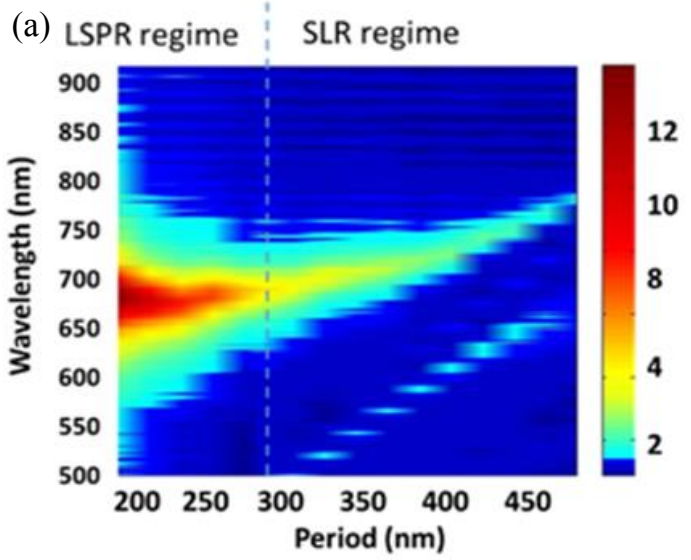

(b)



Fig. 1. (a) Extinction map of the $\mathrm{Ag}$ arrays as a function of the period and wavelength. (b) Variation of the LSP resonance, the first excited photonic mode and the appeared Rayleigh Anomalies as a function of the period.

Depending on the grating period, the obtained results emphasize the existence of two coupling regimes, localized surface plasmon resonance (LSPR) regime [3] and the surface lattice resonance (SLR) regime [4]. The former is associated to short period gratings of $p<$ $280 \mathrm{~nm}$, for which a single resonance characterized by a high damping rate is obtained. In this case, each metallic 
nanoparticle presents intense confined (LSPR) modes. The latter regime is related to inter-particle distance $p \geq$ $280 \mathrm{~nm}$, for which we observe a set of narrow resonances appearing on the extinction spectra that reveals the excitation of collective modes (SLR). Figure 1.b reports the spectral positions of the experimental LSP resonance, the first narrow peak and the associated Rayleigh anomalies [5]. As shown in this figure, the excited sharp resonances closely follow the dispersion of Rayleigh anomalies. Thereby, in this particular case, the coupling between the nanoparticles is mediated via delocalized modes, highly dominated by dipolar far-field effects.

In addition to that, we also performed fluorescence lifetime measurements by using a time correlated single photon counting technique (TCSPC). The obtained results show significant lifetime reductions of the radiative excited states for the short grating periods from $1.57 \mathrm{~ns}$ to $1.09 \mathrm{~ns}$. In contrast to that, no significant reductions have been obtained in the case of the SLR regime according to their delocalized photonic features.

This part of our work shows that, by tuning the nanoparticle grating parameters, it is possible to select specific optical responses for emitters at near or far field zones.

(a)
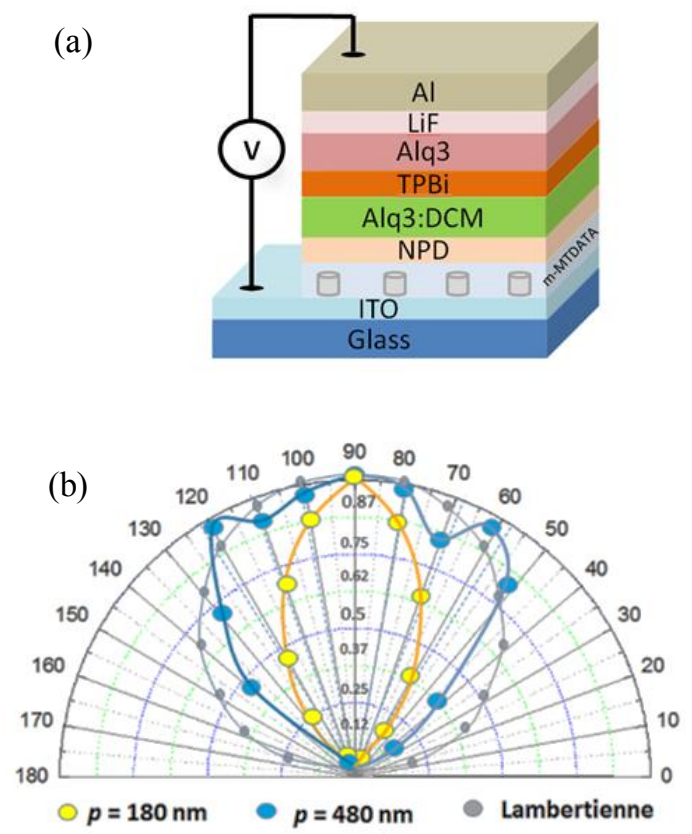

Fig. 2. (a) Scheme of the plasmonic organic light emitting diode. (b) Angular emission diagram for a conventional, plasmonic short and long period Ag-grating based OLEDs.

Based on the previous study, we investigate the plamonic effect of the studied Ag gratings on the optical and electrical properties of OLED devices. The studied OLED is depicted in fig.2.a. As an example, two devices containing gratings of $p=180 \mathrm{~nm}$ and $p=480 \mathrm{~nm}$ have been fabricated and studied (fig.2.a). IVL measurements and angular electro-luminance pattern have been measured and compared to a reference device without nanoparticles.
In addition to the $I V L$ enhancement properties of the plasmonic OLEDs, the obtained emission patterns (fig.2.b) show various features depending on the array period. In the case of the OLED containing the shortperiod grating ( $p=180 \mathrm{~nm})$, the emission is modified to a more directional one, fully contained in a cone aperture of 30 degrees, as compared to the well-known Lambertien pattern of the reference device. However, in the case of the OLED containing a long-period array $(p=480 \mathrm{~nm})$, the pattern shows a maximum of emission in preferred directions, at $\pm 30^{\circ}$ from the normal to the substrate. Therefore, the redirection of the emission can be attributed to the excitation of hybrid modes (SLR); the metallic nanoparticles interacting via a dipole-dipole coupling are involved in constructive interference processes, leading to a significant re-emission of the light into diffractive modes at well-defined directions.

\section{Conclusion}

In this work, we report the investigation of the influence of Ag nanoparticle gratings on the OLED features. Our results demonstrate the existence of two coupling regimes dominated by near or far field effects, respectively. These plasmonic structures can strongly modify the optical and electrical performances of OLED devices. Specifically, the emission pattern can be drastically changed to a more directed one. Therefore, plasmonic structures can be used as an integrated nano-optical compound to spectrally and spatially shape and control the emission in terms of intensity, efficiency and directionality.

\section{References}

1. B. Lamprecht, G. Schider, R. T. Lechner, H. Ditlbacher, J.R. Krenn, A. Leitner, and F. R. Aussenegg, "Metal Nanoparticle Gratings: Influence of Dipolar Particle Interaction on the Plasmon Resonance," PRL, 84 (20), 4721-4724 (2000).

2. T. D. Neal, K. Okamoto, and A. Scherer, "Surface plasmon enhanced emission from dye doped polymer layers," Optics Express , 13(14), 5522-5527 (2005).

3. M. A. Garcia, "Surface plasmon in metallic nanoparticles: fundamentals and applications," Journal of Physics D: Applied Physics, 44(28), 283001-283020 (2011).

4. D. Khlopin, F. Laux, W. P.Wardley, J. Martin, G. A.Wurtz, J. Plain, N. Bonod, A. V. Zayats, W. Dickson and D. Gerard, "Lattice modes and plasmonic linewidth engineering in gold and aluminum nanoparticle arrays," JOSA B, 34(3), 691-700 (2017).

5. A. Maradudin, I. Simonsen, J. Polancoand R. M. Fitzgerald, "Rayleigh and Wood anomalies in the diffraction of light from a perfectly conducting reflection grating," Journal of Optics, 18(2), 024004024014 (2016). 